\title{
万古霉素衍生物一一特拉万星的合成方法改进
}

\author{
刘泺潘 敏周静珊 杨金纬* \\ (浙江医药股份有限公司新昌制药厂 绍兴 312500)
}

\begin{abstract}
摘要 将特拉万星的合成路线进行了工艺改进，通过连续反应方法制得关键中间体 $N$-Fmoc-癸基氨基乙醛，然后经一 锅法及曼尼希反应, 反相硅胶制备得到特拉万星盐酸盐. HPLC 纯度及收率均高于已有文献报道, 且整条路线操作简便, 成本更低, 环境友好, 适于工业化生产.
\end{abstract}

关键词 特拉万星; 万古霉素; 工艺改进

\section{Process Improvement on the Synthesis of Telavancin-Derivative of Vancomycin}

\author{
Liu, Luo Pan, Min Zhou, Jinshan Yang, Jinwei* \\ (Zhejiang Medicine Co., Ltd. Xinchang Pharma, Shaoxing 312500)
}

\begin{abstract}
This article was the process improvement of telavancin synthesis. The key intermediate $N$-Fmoc-decyl-aminoacetaldehyde was obtained through continuous reaction method, followed by one-pot condensation and Mannich reaction. Telavancin hydrochloride was finnally obtained via reversed phase silica gel. The HPLC purity and total yield of this route were higher than the existing literatures. It was easy to operate, environmentally friendly, lower costs, and suitable for industrialized production.
\end{abstract}

Keywords telavancin; vancomycin; process improvement

特拉万星 \{Telavancin，Nvan-2-(癸基氨基)乙基29-[( $\mathrm{N}$-膦酰基甲基)氨甲基]万古霉素(1) $\}$ 是美国施万 (Theravance)公司和日本安斯泰来(Astellas)公司合作开 发的一种静脉使用的新型半合成脂糖肽抗生素. 其注射 剂于 2009 年在美国上市, 被指定用于治疗成人复杂皮 肤感染和皮肤结构感染(cSSSI), 以及由革兰阳性菌引 起的感染, 包括金黄色葡萄球菌(甲氧西林葡萄球菌和 耐甲氧西林葡萄球菌)、化脓性链球菌、无乳链球菌或 粪肠球菌等 ${ }^{[1]}$.

特拉万星是第二代半合成脂糖肽抗生素, 其药物母 核为万古霉素. 在万古霉素糖胺上连接疏水性侧链 (decylaminoethyl), 有利于针向膜、延长半衰期以及增加 对肠球菌的抗菌活力; 而在第 7 位氨基酸的酰基位上连 接了磷酸氨基(phosphonomethyl), 增加了水溶性, 改善 了药物在体内的药动学性质 ${ }^{[2]}$.

对于疏水性侧链 $N$-(9-芴基甲氧羰基)-癸基氨基乙 醛(6)的合成, 原研专利 ${ }^{[3]}$ 以癸醛、乙醇胺为原料, 经还 原氢化反应制得 2-( $N$ - 癸基氨基)乙醇, 再用氯甲酸-9-芴

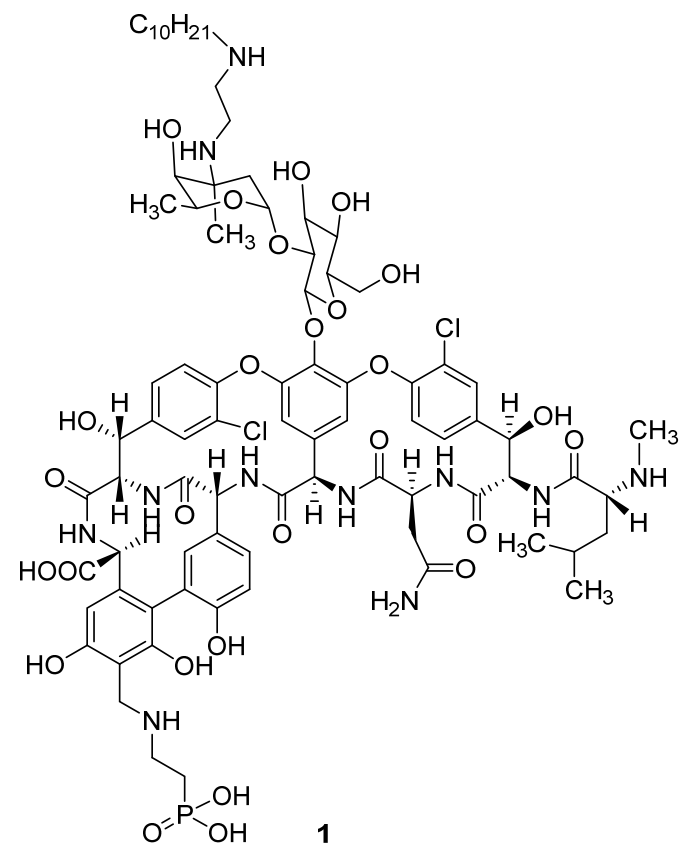

甲酯(Fmoc-Cl)保护亚氨基, 然后经 Swern 氧化反应制

\footnotetext{
* E-mail: yangjw@xcpharma.com

Received May 18, 2015; revised June 18, 2015; published online July 2, 2015.
} 
得. 该反应需用到草酰氯和 DMSO, 反应溶剂需做无水 处理, 环境污染严重, 且在 $-40{ }^{\circ} \mathrm{C}$ 下进行, 反应产物复 杂, 后处理麻烦, 不适于工业化生产; 而梁玉华等 ${ }^{[4]}$ 报 道以癸醛、氨基乙醛缩二甲醇为原料反应先生成席夫碱, 再用氯基硼氢化钠还原得到 $N$-癸基氨基乙醛缩二甲醇 (4), 最后经 Fmoc-Cl 保护亚氨基、酸水解缩醛制得 $N-(9-$ 芴基甲氧羰基)-正癸氨基乙醛(6). 该路线合成 4 的反应 不完全, 有大量原料剩余, 6 的合成用无水 THF 作溶剂, 增加了后处理的复杂性和成本.
本文在参考相关文献 $[3 \sim 8]$ 的基础上，对特拉万星 的合成路线进行了改进. 以正癸胺、乙二醛二甲基缩醛 为原料经席夫碱的还原氢化制得化合物 4 , 再以连续反 应方法经 Fmoc 保护亚氨基、酸水解缩醛得到关键中间 体 $N$-(9-芴基甲氧羰基)-正癸氨基乙醛(6). 6 不经纯化直 接与万古霉素盐酸盐经一锅法制得中间体 $N^{\mathrm{van}}$-2-(正癸 氨基)乙基-万古霉素(8), 再与胺甲基膦酸(9)经曼尼希 反应得到目标化合物 1 . 具体合成路线见 Scheme 1.

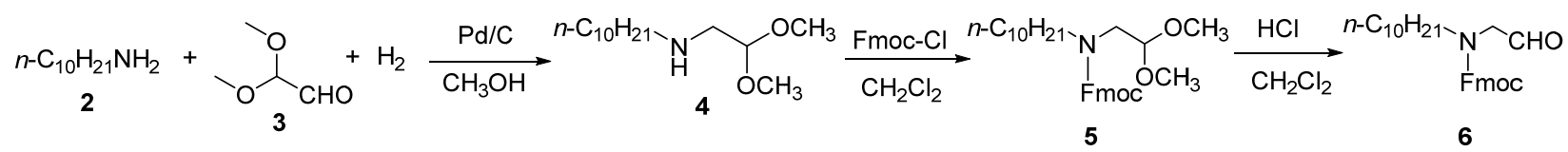

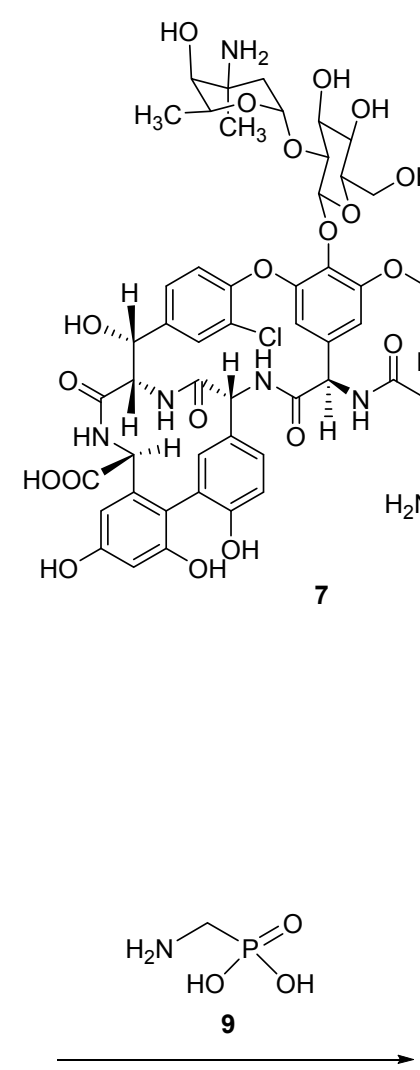

HCHO/DIEA

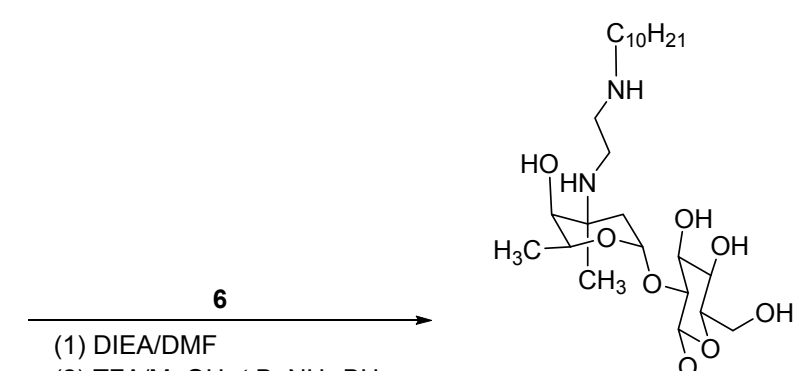

(2) $\mathrm{TFA} / \mathrm{MeOH}, t-\mathrm{BuNH}_{2} \cdot \mathrm{BH}_{3}$ (3) $t-\mathrm{BuNH}_{2}$

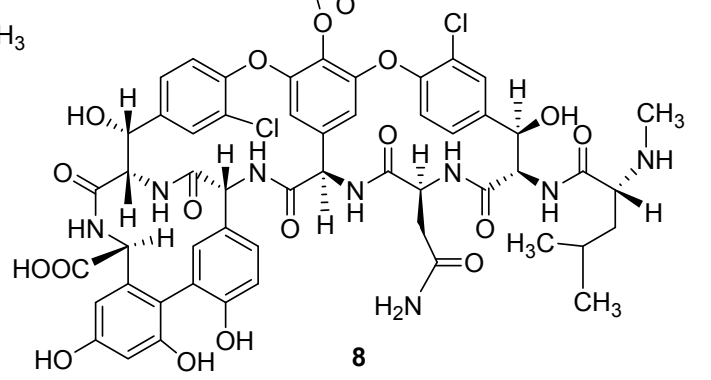

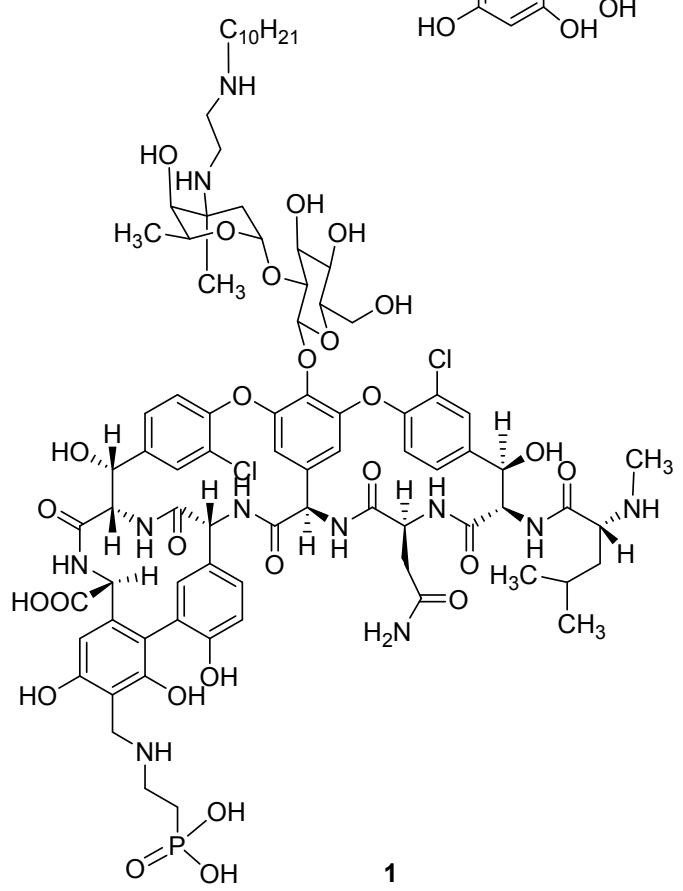

图式 1 特拉万星合成路线

Figure 1 Synthesis of telavancin 


\section{1 结果与讨论}

本文在制备关键中间体 6 时，先经席夫碱的还原氢 化反应制得化合物 4, 收率可达 98\%以上, 产品经浓缩 后可直接进行下一步反应; 后续的 Fmoc 保护亚氨基、 酸水解缩醛反应均在二氯甲烷体系中完成, Fmoc 保护 萃取后的有机相直接加酸水解, 较文献 [3 5]操作更方 便，收率更高，三步收率达到 $89.3 \%$ (文献值 ${ }^{[4]}$ : $82.8 \%$, 文献值 ${ }^{[5]}: 88.0 \%$ ).

化合物 6 无需纯化直接与万古霉素经一锅法制得中 间体 $\mathbf{8}$, 又减少了额外操作; 还原亚胺的反应用了硼烷叔丁胺络合物, 也减少了氭基硼氢化钠之类试剂对环境 的污染; 8 再与胺甲基膦酸(9)经曼尼希反应得到特拉万 星粗品, 粗品经一种新颖的 C-18 反相硅胶柱层析方法

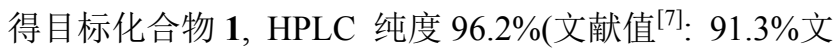
献值 ${ }^{[4]}: 91.8 \%$ 文献值 ${ }^{[5]}: 93.6 \%$ ); 以万古霉素为起始原 料计, 总收率为 $50.4 \%$, 高于文献值 $40.97 \%$ [ ${ }^{[5]}$ 和 $36.3 \%{ }^{[4]}$.

\section{2 实验部分}

\section{1 仪器与试剂}

Agilent 1200 Series 型高效液相色谱仪(美国安捷伦 科技有限公司); BrukerAvance 400 型核磁共振仪(德国 Bruker 公司, TMS 为内标); $1200 \mathrm{HPLC} / 6520$ Q-TOF 型液 质联用仪(美国安捷伦科技有限公司); 正癸胺(梯希爱上 海化成工业发展有限公司); 乙二醛二甲基缩醛(阿法埃 莎天津化学有限公司); Fmoc-Cl(杭州杭试器化玻有限公 司); 嗍烷一叔丁胺络合物(山东威智医药工业有限公司); 叔丁胺(国药集团化学试剂有限公司); 万古霉素盐酸盐 (浙江医药新昌制药厂); 胺甲基膦酸(绍兴英风化工技术 有限公司). 实验所用试剂均为市售分析纯.

\section{2 实验方法}

\subsubsection{N-正癸氨基乙醛缩二甲醇(4)的合成}

室温下, 将 $134.6 \mathrm{~g}$ 正癸胺和 $149.9 \mathrm{~g}$ 乙二醛二甲基 缩醛溶于 $500 \mathrm{~mL}$ 甲醇中，投入 $\mathrm{Pd} / \mathrm{C}$ (含水量 $50 \%$ ) $6.7 \mathrm{~g}$, 将反应液转移至 $1 \mathrm{~L}$ 氢化釜中; 设置氢气压力 $0.8 \mathrm{MPa}$, 体系温度升至 $45{ }^{\circ} \mathrm{C}$, 压力始终保持 $0.8 \mathrm{MPa}$; 反应 $4 \mathrm{~h}$ 后, TLC 跟踪至乙二醛二甲基缩醛消失[展开剂: $V$ (石油 醚) $: V($ 乙酸乙酯 $)=1: 1]$; 过滤 $\mathrm{Pd} / \mathrm{C}$, 减压蒸去甲醇, 往残留物中加入 $1.5 \mathrm{~L} \mathrm{CH}_{2} \mathrm{Cl}_{2}$ 和 $1.5 \mathrm{~L} 2 \mathrm{~mol} / \mathrm{L} \mathrm{HCl}$ 稀释, 分出有机层, 水层再用 $500 \mathrm{~mL} \mathrm{CH}_{2} \mathrm{Cl}_{2}$ 萃取, 合并有机 层, 依次用饱和碳酸氢钠 $(2 \mathrm{~L})$ 以及饱和食盐水 $(2 \mathrm{~L})$ 洗 涤. 有机相经减压浓缩, 得微褐色油状液(214 g, 102\%), 不经纯化直接做下一步反应. ${ }^{1} \mathrm{H}$ NMR (400 MHz, $\mathrm{CDCl}_{3}$ ) $\delta: 4.43(\mathrm{t}, J=5.3 \mathrm{~Hz}, 1 \mathrm{H}), 3.34$ (s, 6H), 2.69 (d,
$J=5.3 \mathrm{~Hz}, 2 \mathrm{H}$ ), 2.55 (t, $J=8.2 \mathrm{~Hz}, 2 \mathrm{H}$ ), 1.43 (brs, 2H), $1.26 \sim 1.21(\mathrm{~m}, 15 \mathrm{H}), 0.83(\mathrm{t}, J=6.8 \mathrm{~Hz}, 3 \mathrm{H})$. HRMS calcd for $\mathrm{C}_{14} \mathrm{H}_{31} \mathrm{NNaO}_{2}(\mathrm{M}+\mathrm{Na})^{+}$268.2252, found 268.2258 .

2.2.2 $\mathrm{N}$-(9-芴基甲氧羰基)-正癸氨基乙醛(6)的合成

将 209.7 g 2-(正癸氨基)乙醛缩二甲醇分散于 800 $\mathrm{mL} \mathrm{CH} \mathrm{Cl}_{2}$ 中, 于冰水浴中冷却至 $10{ }^{\circ} \mathrm{C}$ 以下, 滴加 $110.4 \mathrm{~g}$ DIEA ( $N, N$-二异丙基乙胺), $15 \mathrm{~min}$ 内滴完; 将

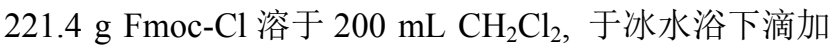
至底物中，控制滴速，使温度不高于 $15^{\circ} \mathrm{C}, 1 \mathrm{~h}$ 滴完; 于 该温度下搅拌 $30 \mathrm{~min}$ 后, TLC 检测 Fmoc- $\mathrm{Cl}$ 反应完全[展 开剂: $V$ (石油醚) $: V($ 乙酸乙酯 $)=3: 1]$; 往反应体系中 加入 $2 \mathrm{~L} 2 \mathrm{~mol} / \mathrm{L} \mathrm{HCl}$ 稀释, 分出有机层, 水层再用 500 $\mathrm{mL} \mathrm{CH}_{2} \mathrm{Cl}_{2}$ 萃取, 合并有机层, 依次用水 $(2 \mathrm{~L})$ 以及饱和 食盐水 $(2 \mathrm{~L})$ 洗涤.

有机相不经浓缩直接滴加 $713 \mathrm{~mL}$ 浓盐酸, $1 \mathrm{~h}$ 滴完, 室温反应 $5 \mathrm{~h}$ 后, TLC 跟踪至底物消失[展开剂: $V($ 石油 醚) $: V($ 乙酸乙酯 $)=3: 1]$; 在反应体系中加 $1 \mathrm{~L}$ 水稀释, 分出有机层, 水层用 $500 \mathrm{~mL} \mathrm{CH}_{2} \mathrm{Cl}_{2}$ 萃取 1 次, 合并有 机层, 依次用饱和碳酸氢钠 $(2 \mathrm{~L})$ 以及饱和食盐水 $(2 \mathrm{~L})$ 洗 涤. 减压蒸去溶剂, 得浅褐色油状液, 不需纯化(388 g, 收率 87.5\%). ${ }^{1} \mathrm{H}$ NMR (400 MHz, $\left.\mathrm{CDCl}_{3}\right) \delta: 9.38$ (d, $J=$ $117.7 \mathrm{~Hz}, 1 \mathrm{H}), 7.76$ (t, $J=6.8 \mathrm{~Hz}, 2 \mathrm{H}), 7.60$ (d, $J=7.4 \mathrm{~Hz}$, $1 \mathrm{H}), 7.51(\mathrm{~d}, J=7.4 \mathrm{~Hz}, 1 \mathrm{H}), 7.44 \sim 7.37(\mathrm{~m}, 2 \mathrm{H}), 7.32$ (dd, $J=11.6,7.3 \mathrm{~Hz}, 2 \mathrm{H}), 4.54$ (dd, $J=8.8,5.9 \mathrm{~Hz}, 2 \mathrm{H})$, 4.21 (ddd, $J=22.7,14.5,5.5 \mathrm{~Hz}, 1 \mathrm{H}), 3.95$ (s, 1H), 3.71 (s, $1 \mathrm{H}), 3.33 \sim 3.18(\mathrm{~m}, 1 \mathrm{H}), 3.18 \sim 3.04(\mathrm{~m}, 1 \mathrm{H}), 1.52 \sim 1.36$ $(\mathrm{m}, 1 \mathrm{H}), 1.37 \sim 1.14(\mathrm{~m}, 14 \mathrm{H}), 1.15 \sim 1.03(\mathrm{~m}, 1 \mathrm{H}), 0.97 \sim$ $0.79(\mathrm{~m}, 3 \mathrm{H}) ;{ }^{13} \mathrm{C} \mathrm{NMR}\left(400 \mathrm{MHz}, \mathrm{CDCl}_{3}\right) \delta: 198.74$, $198.50,156.59,155.64,143.89,143.81,141.44,141.36$, $127.73,127.13,127.09,124.81,124.70,119.99,67.35$, $67.20,57.52,56.92,49.13,48.84,47.33,31.92,29.64$, $29.60,29.55,29.35,28.58,28.13,26.73,26.64,22.72$, 14.17. HRMS: calcd for $\mathrm{C}_{27} \mathrm{H}_{35} \mathrm{NNaO}_{3}(\mathrm{M}+\mathrm{Na})^{+}$ 444.2515, found 444.2518 .

\subsection{3 $N^{\mathrm{van}}$-2-(正癸氨基)乙基-万古霉素 $(8)$ 的合成}

室温下, 将 $958.7 \mathrm{~g}$ 盐酸万古霉素缓慢加到 $6 \mathrm{~L} \mathrm{N,N-}$ 二甲基甲酰胺(DMF)分散, 然后加入 DIEA, 加毕于室 温下搅拌 $15 \mathrm{~min}$; 滴加 $N$-Fmoc-2-(正癸胺基)乙醛(388 g, $80 \%$ ) 的 $2 \mathrm{~L} \mathrm{DMF}$ 溶液, 滴毕室温搅拌 $20 \mathrm{~h}$; 加入 $4 \mathrm{~L}$ 甲 醇以及 $294.1 \mathrm{~g} \mathrm{TFA}$, 搅拌 $30 \mathrm{~min}$ 后加入 $84.1 \mathrm{~g}$ 嗍烷一叔 丁胺络合物, 升温至 $45^{\circ} \mathrm{C}$ 反应 $3 \mathrm{~h}$; 降至室温, 加入叔丁 胺, 升至 $45{ }^{\circ} \mathrm{C}$ 继续反应 $3 \mathrm{~h}$; 在此温度下滴加 $2.5 \mathrm{~L} 1$ $\mathrm{mol} / \mathrm{L} \mathrm{HCl}$, 再慢慢滴加 $10 \% \mathrm{NaCl}$ 溶液 $30 \mathrm{~L}$, 搅拌析出 白色沉淀，降至室温后冷到 $-10{ }^{\circ} \mathrm{C}$ 析晶. 用布氏漏斗抽 
滤, 滤饼用冷水洗涤, 抽干, 得白色固体; 将固体置于 $12 \mathrm{~L}$ 乙腈中打浆, 静置. 抽滤, 滤饼用乙腈洗涤后于 $30{ }^{\circ} \mathrm{C}$ 真空干燥过夜. (991.5 g, HPLC 含量 80\%, 粗收率 $94 \%)$. 不经纯化直接投下一步. HRMS calcd for $\mathrm{C}_{78} \mathrm{H}_{102}-$ $\mathrm{Cl}_{2} \mathrm{~N}_{10} \mathrm{O}_{24}[\mathrm{M}+2 \mathrm{H}]^{2+} 1630.6289$, found 816.3224.

2.2.4 $N^{\mathrm{van}}$-2-(正癸氨基)乙基-29-[( $N$-膦酰甲基)氨甲 基]万古霉素(1)的制备

室温下, 将 $2.7 \mathrm{~g}$ 氨甲基膦酸分散在 $50 \mathrm{~mL} / 50 \mathrm{~mL}$

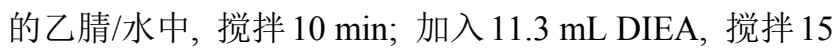
$\mathrm{min}$; 加入 $N^{\mathrm{van}}$-2-(正癸氨基)乙基-万古霉素盐酸盐 $10 \mathrm{~g}$, 室温下摚拌 $1 \mathrm{~h}$ 后将内温将至 $-7{ }^{\circ} \mathrm{C}$, 后加入 $0.44 \mathrm{~mL}$ $37 \%$ 的甲醛, 加毕保持 $-7{ }^{\circ} \mathrm{C}$ 左右搅拌 $12 \mathrm{~h}$, 至 HPLC 检 测脱保护产物含量 $<2.0 \%$; 在此温度下, 用 $6 \mathrm{~mol} / \mathrm{L}$ $\mathrm{HCl}$ 调 $\mathrm{pH}$ 至 2.6 , 升至室温, 往反应液中滴加 $300 \mathrm{~mL}$ 乙腈, 析出不溶物, 搅拌, 静置. 抽滤, 滤饼用乙腈洗 涤, $25{ }^{\circ} \mathrm{C}$ 真空干燥过夜, 得微白色固体 $11.5 \mathrm{~g}$ (HPLC 含 量约 $80 \%$ ). 粗品经反相硅胶制备并后处理后得特拉万 星盐酸盐 $4.6 \mathrm{~g}, \mathrm{HPLC}$ 含量 $96.2 \%$, 收率 53.6\%. ${ }^{1} \mathrm{H}$ NMR $\left(500 \mathrm{MHz}, \mathrm{DMSO}-d_{6}\right) \delta: 9.38$ (brs, $\left.1 \mathrm{H}\right), 9.12$ (s, 1H), 9.01 (s, 1H), 8.98 (brs, 2H), 8.83 (brs, 3H), 8.62 (s, 2H), 7.89 (d, $J=21.5 \mathrm{~Hz}, 1 \mathrm{H}), 7.72$ (brs, 1H), 7.59 (brs, 1H), 7.49 (s, 1H), 7.40 (brs, 1H), 7.21 (s, 1H), 7.14 (d, $J=8.5 \mathrm{~Hz}, 1 \mathrm{H})$, 7.06 (d, $J=8.5 \mathrm{~Hz}, 1 \mathrm{H}), 6.84$ (brs, 1H), 6.60 (s, 1H), 6.57 (brs, 1H), 6.49 (s, 1H), $5.49(\mathrm{~s}, 1 \mathrm{H}), 5.46(\mathrm{~s}, 1 \mathrm{H}), 5.35$ (s, 1H), 5.08 (brs, 1H), 5.01 (brs, 1H), 4.71 (s, 1H), 4.62 (brs, $1 \mathrm{H}), 4.40$ (brs, 1H), 4.30 (t, $J=6.0 \mathrm{~Hz}, 1 \mathrm{H}), 4.26$ (brs, $1 \mathrm{H}$ ), $4.26 \sim 4.31$ (brs, $1 \mathrm{H}), 4.11$ (d, $J=6.0 \mathrm{~Hz}, 1 \mathrm{H}), 3.95$ (brs, $1 \mathrm{H}), 3.81$ (d, $J=11.0 \mathrm{~Hz}, 2 \mathrm{H}), 3.77$ (brs, 1H), 3.65 (s, 1H), $3.56(\mathrm{t}, J=10.0 \mathrm{~Hz}, 4 \mathrm{H}), 3.29(\mathrm{~s}, 1 \mathrm{H}), 3.22(\mathrm{~s}, 2 \mathrm{H}), 3.19$ (s, 2H), 2.94 (brs, 2H), 2.61 (s, 3H), 1.94 (brs, 1H), 1.84 (brs, 1H), 1.68 (brs, 2H), 1.53 (s, 1H), 1.37 (s, 3H), 1.25 (s, $16 \mathrm{H}), 1.11(\mathrm{~d}, J=5.8 \mathrm{~Hz}, 3 \mathrm{H}), 0.92$ (d, $J=5.7 \mathrm{~Hz}, 3 \mathrm{H})$,
$0.85 \sim 0.87(\mathrm{~m}, 6 \mathrm{H}) ;{ }^{13} \mathrm{C}$ NMR $\left(500 \mathrm{MHz}, \mathrm{DMSO}-d_{6}\right) \delta$ : $172.31,169.20,169.12,167.78,167.58,165.86,159.00$, $158.74,158.54,158.23,156.57,155.53,148.26,142.58$, $140.15,136.06,135.16,135.14,131.84,128.44,127.55$, $127.24,127.18,126.55,126.14,120.41,120.05,119.33$, $118.04,116.78,115.67,107.93,106.13,105.56,96.51$, $77.04,76.81,71.60,70.20,69.21,63.17,61.96,61.22$, $59.71,59.32,59.11,56.61,55.14,53.64,46.87,43.12$, $43.01,41.47,40.10,40.01,39.93,39.84,39.76,39.67$, $32.37,32.35,31.26,31.18,28.84,28.74,28.65,28.45$, $25.77,25.49,23.67,22.88,22.14,22.07,18.90,16.84$, 13.94. HRMS calcd for $\mathrm{C}_{80} \mathrm{H}_{108} \mathrm{Cl}_{2} \mathrm{~N}_{11} \mathrm{O}_{27} \mathrm{P}[\mathrm{M}+2 \mathrm{H}]^{2+}$ 1753.6374 , found 877.8266 .

辅助材料 (Supporting Information) 化合物的 ${ }^{1} \mathrm{H}$ $\mathrm{NMR},{ }^{13} \mathrm{C} \mathrm{NMR}$ 谱图. 这些材料可以免费从本刊网站 (http://sioc-journal.cn/)上下载.

\section{References}

[1] Chang, M. H.; Kish, T. D.; Fung, H. B. Clin. Ther. 2010, 32(13), 2160.

[2] Shang, X. Y.; Ruan, L. J. Chin. J. Ant. 2007, 32, 263 (in Chinese). (尚新艳, 阮丽军, 中国抗生素杂志, 2007, 32, 263.)

[3] Lee, J.; Liu, J. WO 03018607, 2003 [Chem. Abstr. 2003, 138, 205347].

[4] Liang, Y. H.; Feng, W. H. Chin. J. Synth. Chem. 2011, 19(4), 550 (in Chinese). (梁玉华, 冯文化, 合成化学, 2011, 19(4), 550.)

[5] Ma, S.; Cao, S. H. Chin. J. New Drugs 2013, 22(23), 2809 (in Chinese). (马帅, 曹胜华，中国新药杂志, 2013, 22(23), 2809.)

[6] Michael, R. L.; Stacy, M. A.; Bettina. B. J. Ant. 2004, 57(5), 326.

[7] Liu, J; Lee, J. US 20050113561, 2005 [Chem. Abstr. 2005, 142, 451926].

[8] Leadbetter, M.; Linsell, M.; Lee, J.; Liu, J. WO 2003029270, 2003 [Chem. Abstr. 2003, 138, 287982].) 